\title{
The DprE1 enzyme, one of the most vulnerable targets of Mycobacterium tuberculosis
}

\author{
Giovanna Riccardi • Maria Rosalia Pasca • \\ Laurent Roberto Chiarelli • Giulia Manina • \\ Andrea Mattevi • Claudia Binda
}

Received: 15 July 2013 /Revised: 26 August 2013 / Accepted: 27 August 2013 /Published online: 14 September 2013

(C) Springer-Verlag Berlin Heidelberg 2013

\begin{abstract}
The re-emergence of tuberculosis in recent years led the World Health Organization (WHO) to launch the Stop TB Strategy program. Beside repurposing the existing drugs and exploring novel molecular combinations, an essential step to face the burden of tuberculosis will be to develop new drugs by identifying vulnerable bacterial targets. Recent studies have focused on decaprenylphosphoryl-D-ribose oxidase (DprE1) of Mycobacterium tuberculosis, an essential enzyme involved in cell wall metabolism, for which new promising molecules have proved efficacy as antitubercular agents. This review summarizes the state of the art concerning DprE1 in terms of structure, enzymatic activity and inhibitors. This enzyme is emerging as one of the most vulnerable target in M. tuberculosis .
\end{abstract}

Keywords Tuberculosis $\cdot$ DprE1 $\cdot$ Antituberculars $\cdot$ Drug target

\section{Introduction}

In the developed countries, for a long time, tuberculosis (TB) was considered a disease of the past as drugs, developed since 1940 , seemed to be effective in the treatment of this infection.

G. Riccardi $(\triangle) \cdot$ M. R. Pasca $~$ L. R. Chiarelli • A. Mattevi •

C. Binda

Department of Biology and Biotechnology "Lazzaro Spallanzani",

University of Pavia, via Ferrata 1, 27100 Pavia, Italy

e-mail: giovanna.riccardi@unipv.it

G. Manina

School of Life Sciences, Swiss Federal Institute of Technology in Lausanne (EPFL), 1015 Lausanne, Switzerland
However, today with about 9 million new TB cases each year and 1.7 million deaths worldwide, this disease is anything but defeated (Dye et al. 2013).

There are three main explanations for the re-emergence of this threat:

(1) The aetiological agent Mycobacterium tuberculosis can change between replicative and dormant states, causing a latent infection that can evolve in active disease when the immune defences drop (Shi et al. 2010). For example, the TB-HIV co-infection alters the equilibrium between dormant and replicative bacilli with a 20 -fold increased risk compared to healthy individuals (Pawlowski et al. 2012). In 2010, in sub-Saharan Africa, $82 \%$ of the TB cases and $71 \%$ of the associated deaths were TB-HIV associated (WHO 2012). Other regions where TB is correlated with HIV are South Asia (India, Thailand, Indonesia and Myanmar) and Eastern Europe (Ukraine and the Russian Federation) (WHO 2012), where poor sanitary conditions and malnutrition considerably contribute to TB susceptibility.

(2) Immigration from countries with high rates of TB infection as well as the social conditions of immigrants are also considered a relevant risk factor for TB comeback (Fenner et al. 2012).

(3) The emergence of M. tuberculosis strains that are multiresistant to the commonly used antitubercular drugs.

In this context of a worldwide serious sanitary problem of TB re-emergence, searching for new and effective drugs/ targets to treat this disease represents an important challenge. Here we review the current knowledge on the recently identified drug target decaprenyl-phosphoribose 2'-oxidase (DprE1) which catalyzes an essential step in mycobacterial cell wall metabolism. 
Tuberculosis: how to address the plight of multi-resistant strains

Multidrug-resistant tuberculosis (MDR-TB), defined as resistance to both isoniazid (INH) and rifampicin poses a great public health concern and it mainly results from the improper usage of antimicrobial drugs and inefficient implementation of control guidelines. Further acquisition of resistance among MDR-TB strains has generated the so-called extensively drug-resistant strains (XDR-TB, defined as MDR-TB with additional resistance to a fluoroquinolone and a second-line injectable agent) (Dye et al. 2013). About $3 \%$ of new and $20 \%$ of previously TB treated patients have MDR-TB, with most cases occurring in Eastern Europe and central Asia. About $9 \%$ of $M$. tuberculosis MDR strains are XDR and 84 countries have now reported at least one XDR-TB case (WHO 2012). 'Completely' or 'totally' drug-resistant M. tuberculosis strains for which there is no effective treatment are increasingly reported, although criteria for 'totally' drug-resistant TB have not been formally defined yet (Walter et al. 2012). Moreover, the presence of dormant bacilli represents a major hurdle to the successful completion of chemotherapy, due to their distinctive recalcitrance to drugs. On December 31, 2012, the U.S. Food and Drug Administration (FDA) approved a new antitubercular drug (bedaquiline) only for adult patients who suffer from MDR-TB, which requires up to 2 years of treatment (Cohen 2013). This drug inhibits the $C$ subunit of essential enzyme ATP synthase (Andries et al. 2005). It is noteworthy that bedaquiline is suspected to be linked with increased mortality in the Phase II randomized trials for the insurgence of cardiac problem (Voelker 2013); consequently, more time will be necessary to obtain clear information about the risks connected with this drug. A comprehensive description of antimycobacterial drugs currently in Phase II/III clinical trials and preclinical phases for TB treatment is well documented (Engohang-Ndong 2012; Zumla et al. 2013).

In recent years, many efforts have been directed towards the discovery of new leads for TB drug development using both target- and cell-based approaches (Cole and Riccardi 2011).

The first strategy, developed by advances in microbial genomics and genetics, involves identification of new essential mycobacterial targets followed by high-throughput screening (HTS) experiments to find a suitable inhibitor, being aware that compounds identified by this screen often may lack activity against whole cells (Payne et al. 2007).

The second approach involves screening of synthetic compounds or natural products for whole cell activity against $M$. tuberculosis, followed by the selection of spontaneous mutants resistant to hit compounds and the identification of mutations by whole genome sequencing (WGS). It is noteworthy that usually, high level of resistance arises from mutations in the drug target gene, while low level resistance is due to uptake defects or drug efflux mechanisms. Once the candidate gene is identified, complementation and knock-out studies will then be carried out for genetic validation of the target, thus paving the way to drug design-oriented molecular and structural studies. In the past, through this strategy, it has been demonstrated that the InhA enzyme, involved in fatty acid biosynthesis, is the target of $\mathrm{INH}$, the frontline drug utilized in TB treatment (Banerjee et al. 1994). It is widely known that INH is a pro-drug activated by KatG enzyme, and by structural studies it was clearly demonstrated that it forms an adduct with the NAD $(\mathrm{H})$ substrate which inhibits InhA (Johnsson and Schultz 1994; Rozwarski et al. 1998). As the majority of INH-resistant clinical strains are mainly due to the emergence of kat $G$ mutants that do not form an INH-NAD adduct, compounds that directly inhibit InhA, avoiding KatG activation, appear promising candidates for the treatment of multi-drug resistant strains (Lu et al. 2010).

Recently, this second strategy has been successfully used for the ATP synthase inhibitor bedaquiline (Andries et al. 2005) and for a new promising drug target, the DprE1 enzyme (Makarov et al. 2009).

\section{DprE1: a hot target for the new millennium}

Cell wall frames and protects mycobacterial cells, being the primary interface with the surrounding environment. Therefore, cell wall biosynthesis is a fundamental process for the mycobacterial survival as highlighted by the fact that genes of this pathway are mostly essential (Sassetti et al. 2003; Carroll et al. 2012). As a consequence, the enzymes involved in this process represent potentially optimal drug targets and, indeed, drugs like isoniazid and ethambutol, recommended by WHO in standard TB treatment regimens, inhibit different steps of the cell wall biosynthesis (Rombouts et al. 2012; Wolucka 2008). In 2009, Makarov et al. described the nitrobenzothiazinones (BTZs; Fig. 1a) as a new class of compounds with high anti-mycobacterial activity. The genetic analyses of M. tuberculosis, M. bovis BCG, and M. smegmatis resistant mutants revealed that DprE1 is the target of BTZs. DprE1 is a decaprenylphosphoryl-D-ribose oxidase, involved in the biosynthesis of decaprenylphosphoryl-D-arabinose (DPA), an essential component of the mycobacterial cell wall (Fig. 1b). DPA is the only known donor of D-arabinofuranosyl residues for the synthesis of arabinogalactan, a basic precursor for the mycobacterial cell wall core (Wolucka 2008). DprE1 is a flavoprotein that in concert with decaprenylphosphoryl 2-ketoribose reductase (DprE2) catalyses the epimerization of decaprenylphosphoryl-D-ribose (DPR) to DPA, via the formation of the intermediate decaprenylphosphoryl 2-keto-ribose (DPX) (Mikusová et al. 2005). In particular, DprE1 catalyzes the oxidation of DPR to DPX, which is reduced to DPA by the NADH-dependent DprE2 enzyme (Fig. 1b). In this context, 
a
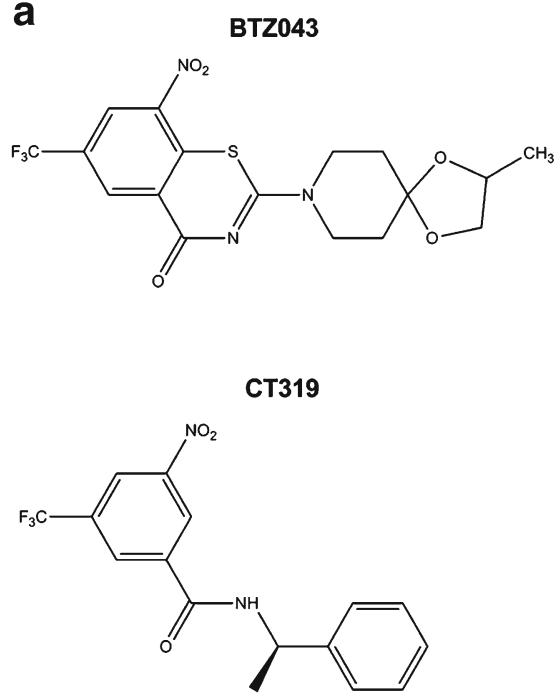

Fig. 1 DprE1 enzymatic function and classes of inhibitors. a Nitroaromatic compounds which covalently inhibit DprE1. BTZ043 in the benzothiazinone class of inhibitors represents the molecule with the most potent antitubercular activity. CT319 is an analog of BTZ043 whose nitroso derivative (CT325) was used for structural characterization. b Scheme showing the FAD-dependent chemical reaction catalyzed by the

b

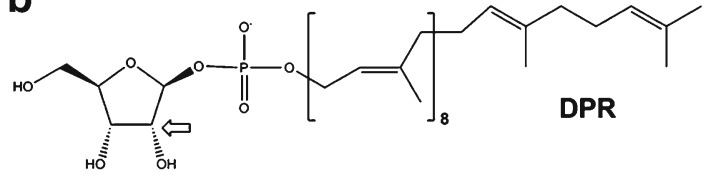

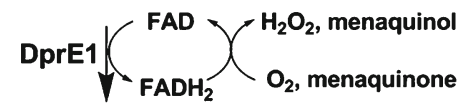<smiles>[R10]CC/C(C)=C/CC/C(C)=C/COP(=O)(OC)O[C@@H]1O[C@H](CO)[C@@H](O)C1=O</smiles>

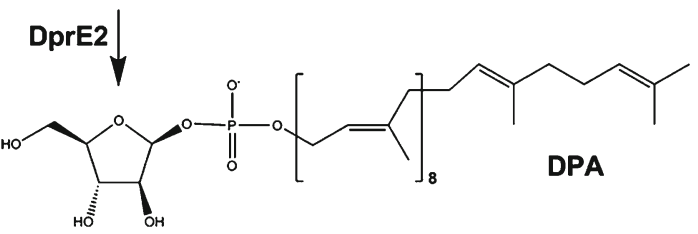

DprE1/DprE2 enzymatic system. The arrow indicates the 2'-OH site of the epimerization reaction of decaprenylphosphoryl-D-ribose $(D P R)$ into decaprenylphosphoryl-D-arabinose $(D P A)$ through the intermediate decaprenylphosphoryl 2-keto-ribose $(D P X)$. During the reaction the FAD cofactor is reduced to $\mathrm{FADH}_{2}$ and can be re-oxidized by either molecular oxygen or organic electron acceptors such as menaquinone
DprE1 was also shown to be essential for cell growth and survival (Crellin et al. 2011).

The most promising BTZ compound (BTZ043; Fig. 1a) is characterized by a nitro group, which seems to be fundamental for effective anti-mycobacterial activity. In fact, two BTZ derivatives, characterized by the nitro group replaced by either an amino or a hydroxylamine group, are much less active (500- to 5,000-fold) (Makarov et al. 2009). Additionally, it has been demonstrated that specific nitroreductases, like the $M$. smegmatis $\mathrm{NfnB}$, are able to inactivate BTZ043, by reducing the nitro group to an amino group. Moreover, it is noteworthy that $M$. tuberculosis lacks $\mathrm{NfnB}$ or NfnB-like enzymes able to inactivate BTZ drugs (Manina et al. 2010).

All M. tuberculosis mutants resistant to BTZ043 were characterized by a point mutation of a cysteine residue of DprE1 (Cys387), as well as mycobacteria naturally resistant to BTZ, such as M. avium and M. aurum, which have a Ser or Gly residue instead of a Cys in their DprE1 enzyme. The collection of these lines of evidence indicated that BTZ043 forms a semimercaptal covalent adduct with DprE1 (Trefzer et al. 2010, 2012).

\section{DprE1: structure and enzymatic characterization}

The recent description of the crystal structures of $M$. tuberculosis and M. smegmatis DprE1 enzymes, in both native form and bound with BTZ or BTZ analogs (Fig. 2) as well as an indepth enzymatic characterization, contributed to a better understanding of this mechanism of inhibition (Batt et al. 2012; Neres et al. 2012). The FAD-dependent oxidation catalyzed by DprE1 implies that the flavin, which is reduced to $\mathrm{FADH}_{2}$ during the reaction, has to be re-oxidized before starting a new catalytic cycle (Fig. 1b). However, despite DprE1 has been always claimed as an oxidase, Neres et al. (2012) showed that the $M$. smegmatis enzyme has a relatively low reactivity with oxygen. In fact, DprE1 can use synthetic electron acceptors such as the 2,6-dichlorophenolindophenol with good efficiency. It was further shown that the reduced enzyme can be efficiently reoxidized by menaquinone, a natural membrane-embedded electron acceptor present in mycobacteria (Fig. 1b), suggesting that DprE1 might be an oxidoreductase, rather than a true oxidase. The same authors showed that BTZ043 is also able to reoxidize in vitro the reduced flavin of DprE1, definitively demonstrating that the nitro group of the drug is effectively converted to a nitroso group by DprE1 itself (Neres et al. 2012). The modified drug is then able to covalently react with the Cys387, leading to covalent inactivation of the enzyme. Recently, Tiwari and collaborators (2013) indicated that BTZ043 might be activated by the cysteine itself because thiolates can induce a non-enzymatic reduction of the nitro group to the corresponding nitroso intermediate by addition at the unsubstituted electron deficient aromatic carbon present in these compounds. These chemical studies offered an alternative hypothesis for the mechanism of action of nitro-aromatic DprE1 inhibitors in that the cysteine thiol(ate) at the active site of DprE1 may trigger the reduction of the nitro groups to the nitroso intermediate, to initiate the inhibition of DprE1. However, the BTZ043-DprE1 covalent adduct formation does not occur in the absence of the substrate (which is required to 

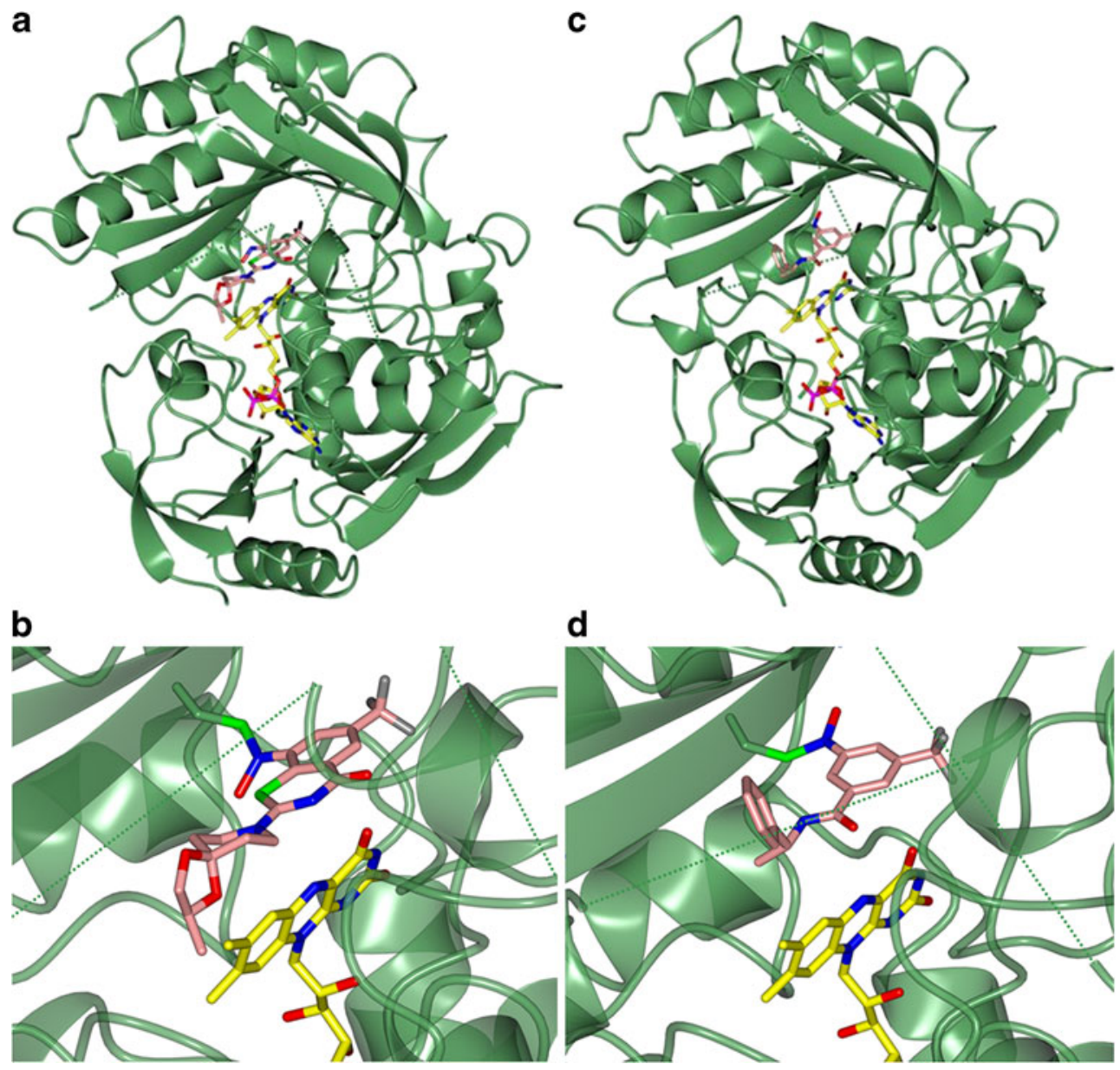

d

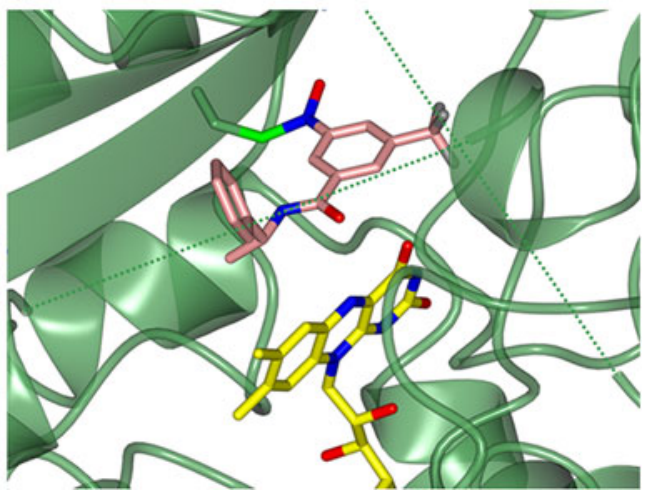

Fig. 2 Three-dimensional structure of DprE1. a Ribbon diagram of the crystal structure of $M$. smegmatis DprE1 in complex with BTZ043 (Fig. 1a) (28) (PDB code 4f4q). The FAD cofactor is depicted with atoms colored as follows: carbon in yellow, nitrogen in blue, oxygen in red, phosphorous in magenta. The BTZ043 molecule is colored as the FAD except for carbon atoms in pink, fluorine atoms in gray, and sulphur atoms in green. Disordered regions (residues 275-303 and 324-336) are represented as dashed lines. b Close-up view of the DprE1-BTZ043 active site structure showing inhibitor binding in more detail with the

covalent linkage to Cys394. Structure orientation and color code are as in panel a. c Crystal structure of M. tuberculosis DprE1 in complex with the nitroso analog of CT319 (Fig. 1a) (27) (PDB code 4fdn). Orientation and color code are as for $M$. smegmatis DprE1 in panels a and $\mathbf{b}$. Disordered regions (residues 269-297 and 315-322) are represented as dashed lines. d Close-up view of the structure shown in panel c, which highlights the inhibitor covalent linkage with Cys387 (corresponding to Cys394 in $M$. smegmatis)

generate $\mathrm{FADH}_{2}$ ), so this hypothesis seems unlikely in the case of DprE1.

The structures of M. smegmatis and M. tuberculosis DprE1 enzymes (which share $83 \%$ sequence identity) are very similar (Fig. 2) (Batt et al. 2012; Neres et al. 2012). Another structure of M. smegmatis DprE1 was recently published which lacks the first 74 residues and does not contain the FAD cofactor ( $\mathrm{Li}$ and Jogl 2013). DprE1 is characterized by the two-domain topology of the vanillyl-alcohol oxidase family of oxidoreductases, such as alditol oxidase, with a FAD-binding domain and a substratebinding domain (Forneris et al. 2008). Differently from alditol oxidase, DprE1 does not bind covalently the prosthetic group. Some active site residues of the two proteins are conserved, consistently with the similarity in the chemistry of their enzymatic activity. Instead, the geometry of the active sites is different, reflecting the nature of the substrate of the two enzymes. Alditol oxidase is characterized by a funnel-like pocket, whereas in the native state DprE1 has an active site cavity in proximity of the protein surface which is shielded from the exterior by a loop formed by residues 322-329 (M. smegmatis numbering) (Neres et al. 2012). This part of the protein is characterized by a certain degree of flexibility as showed by the BTZ043-bound structure in which, beside the disordered portions present also in the native state, residues 324-329 (M. smegmatis) lose their defined conformation (Fig. 2a). This plasticity, which also similarly characterizes the $M$. tuberculosis DprE1 structure, is thought to allow conformational changes of the active site that are probably necessary for the accessibility of the substrate DPR, which, being endowed with a long hydrophobic decaprenyl chain, is likely to be embedded in the cell wall 
and to expose the phosphoribosyl moiety for interaction with DprE1. The hypothesis that DprE1 forms a membrane associated complex with DprE2 is also supported by the fact that the protein surface in proximity of the active site loop is characterized by a cluster of positively charged residues, which might be involved in membrane phospholipids interactions (Batt et al. 2012; Neres et al. 2012).

M. smegmatis DprE1 structure was obtained in the presence of the BTZ043 inhibitor (Neres et al. 2012), while the $M$. tuberculosis homolog was crystallized in complex with CT325 (Batt et al. 2012), the nitroso derivative of CT319 which can covalently react with the active site cysteine (Fig. 1a). The BTZ043-DprE1 complex was reconstituted before crystallization by incubating the protein and the inhibitor with the FPR substrate, in order to have the reduced FAD activating the nitro group of the BTZ043 to form the semimercaptal covalent adduct with Cys394 (Cys387 in $M$. tuberculosis). BTZ043 shares with CT319 (and its nitroso derivative CT325) the nitro-aromatic ring moiety, whereas they totally differ in the other part of the molecule (Fig. 1a). Nevertheless, the inhibitor binding mode is similar in the two structures, with the nitro group covalently linked to the cysteine, though with the oxygen oriented in a different way (Fig. $2 b$ and d). In both structures, the two inhibitors occupy the same position and protrude on the protein surface, with the longer chain of BTZ043 causing displacement of the active site loop (317-329 residues become disordered in $M$. smegmatis DprE1). The active site residues in $M$. smegmatis DprE1 and $M$. tuberculosis DprE1 enzymes are conserved and also their conformation in the inhibitor complex structures are very similar, except for Gln341 and Trp230 whose side chains adopt different conformations.

\section{DprE1: one target, more drugs}

The high 'druggability' of DprE1 is proven by the number of unrelated inhibitors discovered in the last 5 years. Table 1 summarizes the main features of these compounds.

As described above, the first class of compounds is represented by the BTZs. To evaluate the potential clinical development of BTZ resistance, 240 susceptible and multidrugresistant $M$. tuberculosis strains, isolated from four European hospitals, were tested both for BTZ susceptibility and for possible mutations in the $d p r E 1$ gene. All these strains were susceptible giving a solid base for clinical trials (Pasca et al. 2010). The combination of BTZ043 with several antitubercular drugs such as rifampin, isoniazid, ethambutol, TMC207, PA824, moxifloxacin, meropenem with or without clavulanate, and SQ-109 against M. tuberculosis was determined. No antagonism was found and BTZ043 seems to act synergistically with TMC207, suggesting that weakening the cell wall could facilitate the intracellular uptake of other antibacterial molecules (Lechartier et al. 2012).

In order to find new antituberculars, 57,000 small molecules were screened for interfering $M$. tuberculosis replication within macrophages (ex vivo growth). From this screening, 135 not toxic compounds were found to be effective against the pathogen

Table 1 Known compounds targeting DprE1 and their inhibition properties

\begin{tabular}{|c|c|c|c|c|c|}
\hline Compounds & Structure & $\begin{array}{c}\text { MIC in } \\
\text { M. tuberculosis } \\
(\mu \mathrm{M})\end{array}$ & $\begin{array}{l}\text { Nitro- } \\
\text { group }\end{array}$ & $\begin{array}{c}\text { DprE1 } \\
\text { inhibition }\end{array}$ & References \\
\hline BTZ043 & & 0.0023 & yes & covalent & Makarov et al. 2009 \\
\hline DNB1 & & 0.69 & yes & covalent & Christophe et al. 2009 \\
\hline VI-9376 & & 3.1 & yes & covalent & Magnet et al. 2010 \\
\hline 377790 & & $-^{\mathrm{a}}$ & yes & covalent & Stanley et al. 2012 \\
\hline TCA1 & & 0.51 & no & non covalent & Wang et al. 2013 \\
\hline
\end{tabular}

${ }^{\mathrm{a}} \mathrm{The}_{\mathrm{IC}}{ }_{90}$ in M. tuberculosis for this compound is $0.48 \mu \mathrm{M}$ 
and remarkably, among these, the dinitrobenzamide derivatives (DNB) were active also against $M$. tuberculosis XDR strains. The DNBs inhibit the formation of both lipoarabinomannan and arabinogalactan and, more precisely, the DPA synthesis, catalysed by DprE1 enzyme (Christophe et al. 2009). It was found that the BTZ resistant mutants with a mutation in $\operatorname{dprE1}$ gene were also resistant to DNBs, supporting the hypothesis that DprE1 could be also the target of DNBs (Christophe et al. 2009). This hypothesis was finally demonstrated by the isolation of spontaneous M. smegmatis mutants resistant to DNBs and cross-resistant to BTZs with a mutation in $\operatorname{dprE1}$, the same found in BTZ resistant mutants (C394G and C394S) (De Jesus Lopes Ribeiro et al. 2011). In addition, in vitro experiments showed that DNBs directly inhibit DprE1 enzyme activity with inhibition constant similar to that of BTZ043 and forming a covalent adduct with the enzyme with a mechanism analogous to that of the BTZ (Neres et al. 2012).

Novel DprE1-targeting compounds were discovered during the screening of 12,000 compounds to identify new kinase inhibitors effective against both Corynebacterium glutamicum and $M$. tuberculosis by using an integrated strategy involving whole cell-based assays and a target-based approach (Magnet et al. 2010). In this way, 17 compounds non-mutagenic and non-cytotoxic with a minimal inhibitory concentration (MIC) against M. tuberculosis below $10 \mu \mathrm{M}$ were selected. Two of these compounds were effective only against M. tuberculosis and did not inhibit PknA and PknB kinase activity. One of the most active hits, the quinoxaline VI-9376, is structurally related to BTZ compounds. In order to verify whether DprE1 is its target, VI-9376 was tested against M. smegmatis and M. bovis BCG BTZ-resistant mutants with a mutation in this gene. The MIC determination revealed cross-resistance between BTZs and VI-9376; consequently DprE1 could be the target of this compound. The nitro group at the fifth position of the quinoxaline scaffold is absolutely required for the activity (Magnet et al. 2010). Similar to DNB, also the VI-9376 compound was found to inhibit DprE1 in the same manner as BTZ043 (Neres et al. 2012).

Moreover, a recent screen of 341,808 chemical molecules from a NIAID library against $M$. tuberculosis growth had selected some compounds with a good level of activity (Stanley et al. 2012). Resistance to one of the active compounds (377790) was shown to occur due to a mutation in the $d p r E 1$ gene, more precisely the same mutation found in M. tuberculosis BTZ resistant mutants (C387S) (Makarov et al. 2009). The 377790 compound and its derivatives are triazole molecules possessing a nitro group like the BTZ agents. These triazoles are thought to inhibit DprE1 by the formation of a covalent bond via reduction of the nitro group to a nitroso-derivative that reacts specifically with Cys387.

A new molecule, ethyl (2-(benzo[d]thiazole-2-carboxamido) thiophene-3-carbonyl) carbamate (TCA1), active both against replicating and non-replicating $M$. tuberculosis, has been recently published to target DprE1 and MoeW, a protein involved in the biosynthesis of the molybdenum cofactor (Wang et al. 2013). The crystal structure of M. tuberculosis DprE1 in complex with TCA1 reveals that this molecule binds to the enzyme active site in front of the flavin cofactor and establishing a network of hydrophobic and van der Waals interactions with residues which are also involved in BTZ binding. Differently from BTZ043 and CT319 which form a covalent adducts with DprE1, TCA1 molecule does not possess active nitro moiety and the target-binding is mediated by noncovalent interactions (Wang et al. 2013). Although the lack of the nitro group should reduce the possibility of drug inactivation, as is the case of BTZs (Manina et al. 2010), it is noteworthy that at present non covalent drugs resulted less potent than the covalent inhibitors. The identification of new DprE1-targeting compounds with different mechanism of action will pave the way to the development of novel and more effective drugs.

\section{Concluding remarks}

Despite some good progress in TB drug development, much more is urgently needed to face the challenge of multi-drug resistant strains, as there is a worrying gap between the last stage of preclinical studies and the first phase of clinical trials (Zumla et al. 2013). The recently identified M. tuberculosis target DprE1 shed a new light in the strategies to defeat this plague. The druggability of DprE1 is linked to its essential function in mycobacterial cells where this enzyme is fundamental for the construction of the mycobacterial cell wall core. Indeed, DprE1 has become the hottest target: in the last 4 years, five molecules, belonging to different chemical classes, have been published and most likely others are under investigation. At present, BTZ043 with an in vitro MIC of $1 \mathrm{ng} / \mathrm{ml}$ (defined as the lowest concentration that blocked growth of $99 \%$ of the mycobacterial cells) appears as the most potent drug affecting DprE1, even if its in vivo efficacy, in TB murine models, was less evident, perhaps due to the action of an NfnB-like activity (Manina et al. 2010). The structural and biochemical characterization of DprE1 will now guide drug design approaches to optimize the inhibitors and, in particular, to identify new molecular scaffolds targeting this enzyme, whose studies are proving to be the real Achilles' heel of M. tuberculosis .

In recent years, extensive research has been made in drugs targeting M. tuberculosis DprE1, so it is foreseeable that at least one of these drugs could enter clinical trials, which would greatly increase the chances of survival of patients affected from multi-drug resistant TB.

Acknowledgments This work was supported by European Commission (VII Framework, contract no. 260872). 


\section{References}

Andries K, Verhasselt P, Guillemont J, Göhlmann HW, Neefs JM, Winkler H, Van Gestel J, Timmerman P, Zhu M, Lee E, Williams P, de Chaffoy D, Huitric E, Hoffner S, Cambau E, Truffot-Pernot C, Lounis N, Jarlier V (2005) A diarylquinoline drug active on the ATP synthase of Mycobacterium tuberculosis. Science 307: 223-227

Banerjee A, Dubnau E, Quemard A, Balasubramanian V, Um KS, Wilson T, Collins D, de Lisle G, Jacobs WR Jr (1994) inhA, a gene encoding a target for isoniazid and ethionamide in Mycobacterium tuberculosis. Science 263:227-230

Batt SM, Jabeen T, Bhowruth V, Quill L, Lund PA, Eggeling L, Alderwick LJ, Fütterer K, Besra GS (2012) Structural basis of inhibition of Mycobacterium tuberculosis DprE1 by benzothiazinone inhibitors. Proc Natl Acad Sci U S A 109:11354-11359

Carroll P, Faray-Kele MC, Parish T (2012) Identifying vulnerable pathways in Mycobacterium tuberculosis by using a knockdown approach. Appl Environ Microbiol 7:5040-5043

Christophe T, Jackson M, Jeon HK, Fenistein D, Contreras-Dominguez M, Kim J, Genovesio A, Carralot JP, Ewann F, Kim EH, Lee SY, Kang S, Seo MJ, Park EJ, Skovierová H, Pham H, Riccardi G, Nam JY, Marsollier L, Kempf M, Joly-Guillou ML, Oh T, Shin WK, No Z, Nehrbass U, Brosch R, Cole ST, Brodin P (2009) High content screening identifies decaprenyl-phosphoribose $2^{\prime}$ epimerase as a target for intracellular antimycobacterial inhibitors. PLoS Pathog 5:e1000645. doi:10.1371/journal.ppat.1000645

Cohen J (2013) Infectious disease. Approval of novel TB drug celebrated - with restraint. Science 339:130

Cole ST, Riccardi G (2011) New tuberculosis drugs on the horizon. Curr Opin Microbiol 14:570-576

Crellin PK, Brammananth R, Coppel RL (2011) Decaprenylphosphoryl- $\beta$ D-ribose 2'-epimerase, the target of benzothiazinones and dinitrobenzamides, is an essential enzyme in Mycobacterium smegmatis. PLoS One 6:e16869. doi:10.1371/journal.pone.0016869

De Jesus Lopes Ribeiro AL, Degiacomi G, Ewann F, Buroni S, Incandela ML, Chiarelli LR, Mori G, Kim J, Contreras-Dominguez M, Park YS, Han SJ, Brodin P, Valentini G, Rizzi M, Riccardi G, Pasca MR (2011) Analogous mechanisms of resistance to benzothiazinones and dinitrobenzamides in Mycobacterium smegmatis. PLoS One 6:e26675. doi:10.1371/journal.pone.0026675

Dye C, Glaziou P, Floyd K, Raviglione M (2013) Prospects for tuberculosis elimination. Annu Rev Public Health 34:271-286. doi:10. 1146/annurev-publhealth-031912-114431

Engohang-Ndong J (2012) Antimycobacterial drugs currently in Phase II clinical trials and preclinical phase for tuberculosis treatment. Expert Opin Investig Drugs 21:1789-1800

Fenner L, Gagneux S, Helbling P, Battegay M, Rieder HL, Pfyffer GE, Zwahlen M, Furrer H, Siegrist HH, Fehr J, Dolina M, Calmy A, Stucki D, Jaton K, Janssens JP, Stalder JM, Bodmer T, Ninet B, Böttger EC, Egger M, Swiss HIV Cohort Study Group, Molecular Epidemiology of Tuberculosis Study Group (2012) Mycobacterium tuberculosis transmission in a country with low tuberculosis incidence: role of immigration and HIV infection. J Clin Microbiol 50: 388-395

Forneris F, Heuts DP, Delvecchio M, Rovida S, Fraaije MW, Mattevi A (2008) Structural analysis of the catalytic mechanism and stereoselectivity in Streptomyces coelicolor alditol oxidase. Biochemistry 47:978-985

Johnsson K, Schultz PG (1994) Mechanistic studies of the oxidation of isoniazid by the catalase peroxidase from Mycobacterium tuberculosis. J Am Chem Soc 116:7425-7426

Lechartier B, Hartkoorn RC, Cole ST (2012) In vitro combination studies of benzothiazinone lead compound BTZ043 against Mycobacterium tuberculosis. Antimicrob Agents Chemother 56:5790-5793
Li H, Jogl G (2013) Crystal structure of decaprenylphosphoryl- $\beta$-Dribose 2'-epimerase from Mycobacterium smegmatis. Proteins 81: 538-543

Lu XY, You QD, Chen YD (2010) Recent progress in the identification and development of InhA direct inhibitors of Mycobacterium tuberculosis. Mini Rev Med Chem 10:181-192

Magnet S, Hartkoorn RC, Székely R, Pató J, Triccas JA, Schneider P, Szántai-Kis C, Orfi L, Chambon M, Banfi D, Bueno M, Turcatti G, Kéri G, Cole ST (2010) Leads for antitubercular compounds from kinase inhibitor library screens. Tuberculosis (Edinb) 90:354-360

Makarov V, Manina G, Mikusova K, Möllmann U, Ryabova O, SaintJoanis B, Dhar N, Pasca MR, Buroni S, Lucarelli AP, Milano A, De Rossi E, Belanova M, Bobovska A, Dianiskova P, Kordulakova J, Sala C, Fullam E, Schneider P, McKinney JD, Brodin P, Christophe T, Waddell S, Butcher P, Albrethsen J, Rosenkrands I, Brosch R, Nandi V, Bharath S, Gaonkar S, Shandil RK, Balasubramanian V, Balganesh T, Tyagi S, Grosset J, Riccardi G, Cole ST (2009) Benzothiazinones kill Mycobacterium tuberculosis by blocking arabinan synthesis. Science 324:801-804

Manina G, Bellinzoni M, Pasca MR, Neres J, Milano A, Ribeiro AL, Buroni S, Skovierová $\mathrm{H}$, Dianišková $\mathrm{P}$, Mikušová $\mathrm{K}$, Marák $\mathrm{J}$, Makarov V, Giganti D, Haouz A, Lucarelli AP, Degiacomi G, Piazza A, Chiarelli LR, De Rossi E, Salina E, Cole ST, Alzari PM, Riccardi G (2010) Biological and structural characterization of the Mycobacterium smegmatis nitroreductase $\mathrm{NfnB}$, and its role in benzothiazinone resistance. Mol Microbiol 77:1172-1185

Mikusová K, Huang H, Yagi T, Holsters M, Vereecke D, D'Haeze W, Scherman MS, Brennan PJ, McNeil MR, Crick DC (2005) Decaprenylphosphorylarabinofuranose, the donor of the Darabinofuranosyl residues of mycobacterial arabinan, is formed via a two-step epimerization of decaprenylphosphoryl ribose. J Bacteriol 187:8020-8025

Neres J, Pojer F, Molteni E, Chiarelli LR, Dhar N, Boy-Röttger S, Buroni S, Fullam E, Degiacomi G, Lucarelli AP, Read RJ, Zanoni G, Edmondson DE, De Rossi E, Pasca MR, McKinney JD, Dyson PJ, Riccardi G, Mattevi A, Cole ST, Binda C (2012) Structural basis for benzothiazinone-mediated killing of Mycobacterium tuberculosis. Sci Transl Med 4:150ra121. doi:10.1126/scitranslmed.3004395

Pasca MR, Degiacomi G, Ribeiro AL, Zara F, De Mori P, Heym B, Mirrione M, Brerra R, Pagani L, Pucillo L, Troupioti P, Makarov V, Cole ST, Riccardi G (2010) Clinical isolates of Mycobacterium tuberculosis in four European hospitals are uniformly susceptible to benzothiazinones. Antimicrob Agents Chemother 54:1616-1618

Pawlowski A, Jansson M, Sköld M, Rottenberg ME, Källenius G (2012) Tuberculosis and HIV co-infection. PLoS Pathog 8:e1002464. doi: 10.1371/journal.ppat.1002464

Payne DJ, Gwynn MN, Holmes DJ, Pompliano DL (2007) Drugs for bad bugs: confronting the challenges of antibacterial discovery. Nat Rev Drug Discov 6:29-40

Rombouts Y, Brust B, Ojha AK, Maes E, Coddeville B, Elass-Rochard E, Kremer L, Guerardel Y (2012) Exposure of mycobacteria to cell wall-inhibitory drugs decreases production of arabinoglycerolipid related to mycolyl-arabinogalactan-peptidoglycan metabolism. J Biol Chem 287:11060-11069

Rozwarski DA, Grant GA, Barton DH, Jacobs WR Jr, Sacchettini JC (1998) Modification of the NADH of the isoniazid target (InhA) from Mycobacterium tuberculosis. Science 279:98-102

Sassetti CM, Boyd DH, Rubin EJ (2003) Genes required for mycobacterial growth defined by high density mutagenesis. Mol Microbiol 48: 77-84

Shi L, Sohaskey CD, Pfeiffer C, Datta P, Parks M, McFadden J, North RJ, Gennaro ML (2010) Carbon flux rerouting during Mycobacterium tuberculosis growth arrest. Mol Microbiol 78:1199

Stanley SA, Grant SS, Kawate T, Iwase N, Shimizu M, Wivagg C, Silvis M, Kazyanskaya E, Aquadro J, Golas A, Fitzgerald M, Dai H, Zhang L, Hung DT (2012) Identification of novel inhibitors of $M$. 
tuberculosis growth using whole cell based high-throughput screening. ACS Chem Biol 7:1377137-1377184

Tiwari R, Moraski GC, Krchňák V, Miller PA, Colon-Martinez M, Herrero E, Oliver AG, Miller MJ (2013) Thiolates chemically induce redox activation of BTZ043 and related potent nitro aromatic anti-tuberculosis agents. J Am Chem Soc 135:3539-3549

Trefzer C, Rengifo-Gonzalez M, Hinner MJ, Schneider P, Makarov V, Cole ST, Johnsson K (2010) Benzothiazinones: prodrugs that covalently modify the decaprenylphosphoryl- $\beta$-D-ribose 2 -epimerase DprE1 of Mycobacterium tuberculosis. J Am Chem Soc 132: 13663-13665

Trefzer C, Škovierová H, Buroni S, Bobovská A, Nenci S, Molteni E, Pojer F, Pasca MR, Makarov V, Cole ST, Riccardi G, Mikušová K, Johnsson K (2012) Benzothiazinones are suicide inhibitors of mycobacterial decaprenylphosphoryl- $\beta$-D-ribofuranose 2 '-oxidase DprE1. J Am Chem Soc 134:912-915

Voelker R (2013) MDR-TB has new drug foe after fast-track approval. JAMA 309:430
Walter ND, Strong M, Belknap R, Ordway DJ, Daley CL, Chan ED (2012) Translating basic science insight into public health action for multidrug- and extensively drug-resistant tuberculosis. Respirology 17:772-791

Wang F, Sambandan D, Halder R, Wang J, Batt SM, Weinrick B, Ahmad I, Yang P, Zhang Y, Kim J, Hassani M, Huszar S, Trefzer C, Ma Z, Kaneko T, Mdluli KE, Franzblau S, Chatterjee AK, Johnson K, Mikusova K, Besra GS, Fütterer K, Jacobs WR Jr, Schultz PG (2013) Identification of a small molecule with activity against drug-resistant and persistent tuberculosis. Proc Natl Acad Sci U S A 110:E2510-E2517. doi:10.1073/pnas.1309171110

WHO, World Health Organization (2012). http://www.who.int/en/

Wolucka BA (2008) Biosynthesis of D-arabinose in mycobacteria - a novel bacterial pathway with implications for antimycobacterial therapy. FEBS J 275:2691-2711

Zumla A, Nahid P, Cole ST (2013) Advances in the development of new tuberculosis drugs and treatment regimens. Nat Rev Drug Discov 12:388-404. doi:10.1038/nrd4001 